\title{
Finite Temperature LGT in a Finite Box with BPS Monopole Boundary Conditions *
}

\author{
E.-M. Ilgenfritz ${ }^{\mathrm{a}}$, S. V. Molodtsov ${ }^{\mathrm{b}}$, M. Müller-Preussker ${ }^{\mathrm{c}}$, and A. I. Veselov ${ }^{\mathrm{b}}$ \\ anstitute for Theoretical Physics, Kanazawa University, Kanazawa 920-1192, Japan \\ bITEP, B. Cheremushkinskaya 25, Moscow 117259, Russia \\ ${ }^{\mathrm{c} I n s t i t u t ~ f u ̈ r ~ P h y s i k, ~ H u m b o l d t-U n i v e r s i t a ̈ t ~ z u ~ B e r l i n, ~ G e r m a n y ~}$
}

Finite temperature $S U(2)$ lattice gauge theory is investigated in a $3 D$ cubic box with fixed boundary conditions (b.c.) provided by a discretized, static BPS monopole solution with varying core scale $\mu$. For discrete $\mu$-values we find stable classical solutions either of electro-magnetic ('dyon') or of purely magnetic type inside the box. Near the deconfinement transition we study the influence of the b.c. on the quantized fields inside the box. In contrast to the purely magnetic background field case, for the dyon case we observe confinement for temperatures above the usual critical one.

\section{INTRODUCTION}

Quark confinement in QCD is a long-standing problem which has not found a satisfactory explanation until now. Several models have been considered. The dual superconductor scenario [1] views confinement as a dual Meissner effect due to the condensation of Abelian monopoles. But it badly serves as a frame for phenomenological applications. On the other hand the instantonbased semi-classical approach has been very successful in phenomenology. However, it has not yet explained confinement. The question arises, whether other extended, maybe monopole-like, semi-classical configurations may support confinement. In this talk we would like to give a partial answer to this question.

The configuration we want to start from is the Bogomol'nyi-Prasad-Sommerfield (BPS) monopole [2] taken as a static solution of the equation of motion in Euclidean $S U(2)$ Yang-Mills theory. Following an investigation reported by Smit and van der Sijs [3] we discretize it on the lattice and keep the tangential boundary values fixed for the 3-volume. With these b.c. we find stable solutions inside the box. Depending on the core scale there are configurations, which can be either

\footnotetext{
${ }^{*}$ Talk given by. A. I. Veselov
}

of purely magnetic type - t'Hooft-Polyakov-like (HP) monopoles [4] - or of self-dual BPS-type. The latter possess also electric charge. Therefore, let us call them 'dyons'. With the stable solutions taken as a well-defined semi-classical background we investigate Monte Carlo generated quantum fields.

For the finite temperature case we shall see, how usual indicators of the deconfinement phase transition (average Polyakov line, distribution of Polyakov lines) behave in the presence of the different background fields.

\section{CLASSICAL FIELDS WITH DYON BOUNDARY CONDITIONS}

Let us discuss classical field configurations on a hypercubic lattice in a finite volume with fixed spatial b.c. The latter will be specified such that link variables being normal to the boundary and pointing outward are inactive, i.e. cannot contribute to the action. Link variables tangential to the boundaries will be fixed to classical values given by a BPS dyon configuration. For the imaginary time direction periodicity is implied. We discretize the BPS dyon field as in [3]. Its center be fixed at the center of the $3 D$ box. The large distance asymptotic behaviour of the BPS dyon gauge potentials provides a periodic behaviour of 
the boundary link variables as a function of the core scale-size parameter $\mu$. It allows to create b.c. compatible either with a BPS dyon or with a HP monopole with suppressed electric fields. Following [3] it is convenient to parametrize the boundary values by an auxiliary parameter $\mu^{\prime}$ instead of $\mu, \quad \mu^{\prime}=\left(\mu-1 / R_{e f f}\right) \cdot N_{t} /(2 \pi)$, where $R_{\text {eff }} \simeq 1.13 \cdot\left(N_{s}-1\right) / 2$.

We wanted to find out, what lattice fields really have minimal action for given b.c. It turned out that there exist several local minima of action for $\mu^{\prime}$ sufficiently big.

In order to characterize the lattice field configurations obtained by heating and cooling we used the full Wilson plaquette action $S=E^{2}+B^{2}$, with its magnetic part $B^{2}$ coming from the spatial plaquette contributions and its electric part $E^{2}$ obtained from the time-like plaquettes.

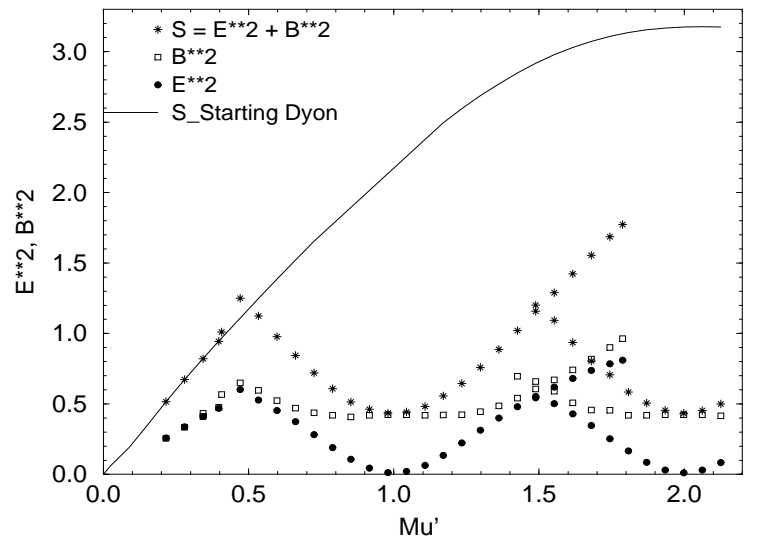

Figure 1. Total action, magnetic and electric part of the action.

Fig. 1 shows the full action per time slice of the classical continuum BPS dyon in dependence on the parameter $\mu^{\prime}$ (solid line). The action values are given in units of $4 \pi /\left(a g^{2}\right)$. The data points in Fig. 1 show the global or local minima, respectively, found for the full action (asterisks) after different attemps of heating and subsequent cooling. The most interesting for us are the states obtained for half-integer and integer $\mu^{\prime}$-values, respectively. For $\mu^{\prime} \simeq 0.5,1.5, \cdots$ we reproduce a BPS dyon state identical to the original one at $\mu^{\prime}=0.5$ with equal electric and magnetic contributions to the action. For $\mu^{\prime} \simeq 1.0,2.0, \cdots$ only the magnetic part of the action survives. That means that we have obtained purely magnetic, HP-like monopoles.

\section{QUANTUM FIELDS WITH DYON BOUNDARY CONDITIONS}

In the following we want to investigate quantum fields for well-defined background fields being either BPS dyon or HP monopole classical lattice solutions depending on the spatial b.c. The simulations have been done with a standard Metropolis algorithm on a $16^{3} \times 4$ lattice. For $N_{t}=4$ the deconfinement phase transition occurs at $\beta=\beta_{c} \simeq 2.29$ in the large-volume limit. In the following we will mainly consider two typical cases: $\beta=2.2$, characteristic for the confinement phase, and $\beta=2.4$, for the deconfinement phase.

By computing space-like and time-like average plaquettes as a function of the distance $d$ from the spatial boundary one can define a core inside the three-volume $(d \geq 3)$, where these local observables take on a plateau value.

We have computed the average Polyakov line as a function of the distance $d$ from the boundary. Even inside the core (at $d \geq 3$ ) the behaviour of the Polyakov line strongly depends on the b.c., i.e. whether there is a BPS dyon or a HP monopole background field. For integer $\mu^{\prime}$ (HP monopole) in both the confinement and the deconfinement phase the average Polyakov line approaches the value characteristic for this $\beta$ (with periodic b.c.). On the contrary, for halfinteger $\mu^{\prime}$ (BPS dyon) the average Polyakov line remains very small throughout the whole lattice when passing the deconfinement transition. At $\beta=2.4$ inside the core we find $|<L>|<0.1$.

These observations are supported by the corresponding histograms of local Polyakov line values measured at distance $d=5$ from the boundary which are shown in Fig. 2 for $\beta=2.2$ and 2.4, respectively. In the confinement phase, the histograms corresponding to the monopole b.c. are displaced representing entirely the effect of the finite correlation length of the Polyakov line, while the histogram for the dyonic b.c. coincides with 
$\beta=2.2$

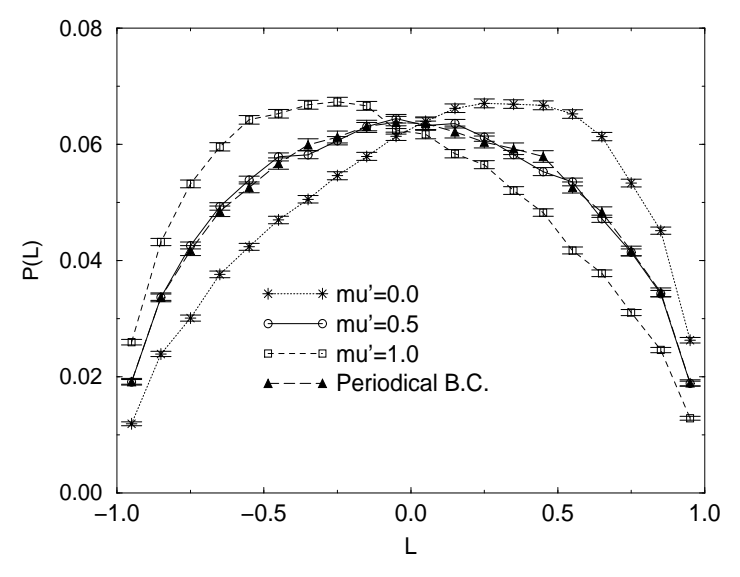

$\beta=2.4$

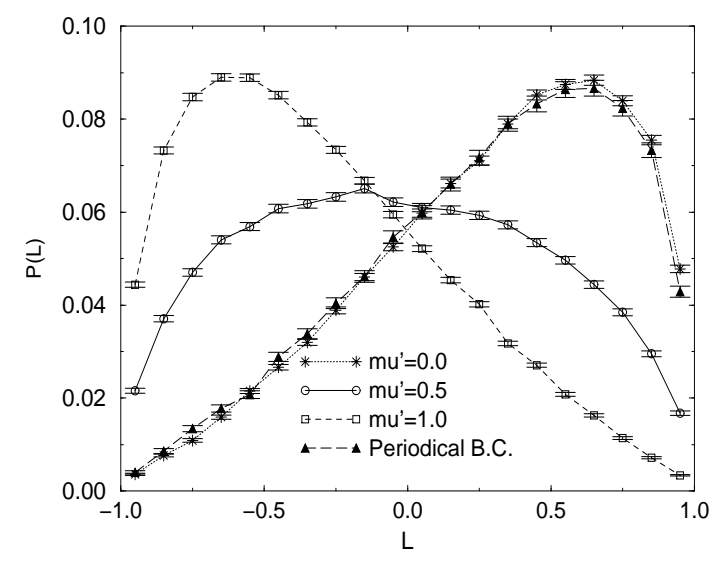

Figure 2. Distributions of local Polyakov line values for various b.c.

that for periodic b.c. In the deconfinement phase the "normal" histogram (generated by staying within one of the possible $Z(2)$-states with periodic b.c.) is reproduced with monopole b.c. while the histogram for dyonic b.c. is almost symmetric, similar to the lower $\beta$-value.

We conclude that, while the HP monopole b.c. are compatible also with the confinement phase, a BPS dyon background inside the finite box keeps the system in the confinement state even for temperatures above $T_{c}$. But as we have seen for smaller $N_{t}$ or larger $\beta$, this does not persist at arbitrarily high temperature.

\section{CONCLUSIONS}

Our main observation was that in the dyon background field case the quantum system inside a finite $3 D$ box is kept in the confinement state even for higher temperatures, where for periodic boundary conditions deconfinement is caused. The Polyakov line as the main order parameter for the deconfinement transition turned out to be zero remarkably stable inside the whole $3 D$ volume considered. However, a further increase of the temperature finally restores deconfinement inside the $3 D$ box. The phenomenon reported here might be called evaporation delay of confinement due to dyon boundary conditions.

We would like to interprete our findings in more general terms that the confinement phenomenon is strongly related to self-dual semiclassical objects rather than to purely magnetic background fields. On the other hand, deconfinement, when described semi-classically, requires background fields which are not (anti-)self-dual.

\section{ACKNOWLEDGEMENTS}

The authors are grateful to P. van Baal, B.V. Martemyanov, M.I. Polikarpov, A.J. van der Sijs, Yu.A. Simonov, and J. Smit for useful discussions. This work was partly supported by RFBR grants 97-02-17491 and 96-02-17230, INTAS-RFBR 950681 and INTAS 96-370 and the joint DFG-RFFI grant 436 RUS 113/309/0 (R) or RFBR-DFG grant 96-02-0088G.

\section{REFERENCES}

1. G. 't Hooft, in High Energy Physics, Proc. EPS Intern. Conf., ed. A. Zichichi, 1976; Nucl. Phys. B190 (1981) 455; S. Mandelstam, Phys. Rep. 23 (1976) 245.

2. M.K. Prasad and C.M. Sommerfield, Phys. Rev. Lett. 35 (1975) 760; E.B. Bogomol'nyi, Sov. J. Nucl. Phys. 24 (1976) 449.

3. J. Smit and A. van der Sijs, Nucl. Phys. B422 (1994) 349.

4. G. 't Hooft, Nucl. Phys. B79 (1974) 276;

A.M. Polyakov, JETP Letters 20 (1974) 276. 